\title{
Insulin-Like Growth Factor 1 Mitigates Hematopoietic Toxicity after Lethal Total Body Irradiation
}

\author{
Dunhua Zhou, MD, PhD ${ }^{1, \#}$, Divino Deoliveira, PhD 1 , Yubin Kang, MD ${ }^{1, \&}$, Seung S. Choi ${ }^{1}$, \\ Zhiguo Li, PhD ${ }^{3}$, Nelson J. Chao, MD ${ }^{1,2,4,5}$, and Benny J. Chen, MD ${ }^{1,5,{ }^{*}}$ \\ ${ }^{1}$ Department of Medicine, Duke University Medical Center, Durham, North Carolina, USA \\ 2Department of Pathology, Duke University Medical Center, Durham, North Carolina, USA \\ ${ }^{3}$ Department of Biostatistics and Bioinformatics, Duke University Medical Center, Durham, North \\ Carolina, USA \\ ${ }^{4}$ Department of Immunology, Duke University Medical Center, Durham, North Carolina, USA \\ ${ }^{5}$ Duke Cancer Institute, Duke University Medical Center, Durham, North Carolina, USA
}

\begin{abstract}
Purpose-To investigate whether and how insulin-like growth factor 1 (IGF-1) mitigates hematopoietic toxicity after total body irradiation.
\end{abstract}

Methods and Materials-BALB/c mice were irradiated with a lethal dose of radiation (7.5 Gy) and treated with IGF-1 at a dose of $100 \mu \mathrm{g} /$ dose intravenously once a day for five consecutive days starting within one hour post exposure. Survival and hematopoietic recovery were monitored. The mechanisms by which IGF-1 promotes hematopoietic recovery were also studied using an in vitro culture system.

Results-IGF-1 protected 8 out of 20 mice (40\%) from lethal irradiation while only 2 out of 20 mice $(10 \%)$ in the saline control group survived for more than 100 days after irradiation. A single dose of IGF-1 $(500 \mu \mathrm{g})$ was as effective as daily dosing for five days. Positive effects were noted even when the initiation of treatment was delayed up to six hours post irradiation. Compared with the saline control group, treatment with IGF-1 significantly accelerated the recovery of both platelets and red cells in peripheral blood, total cell numbers as well as hematopoietic stem cells and progenitors in the bone marrow when measured at day 14 post-irradiation. IGF-1 protected both hematopoietic stem cells and progenitors from radiation-induced apoptosis and cell death. In addition, IGF-1 was able to facilitate the proliferation and differentiation of non-irradiated and irradiated hematopoietic progenitors.

\footnotetext{
(C) 2012 Elsevier Inc. All rights reserved.

*Corresponding author: Benny J. Chen, Division of Cellular Therapy/BMT, Department of Medicine, Duke University Medical Center, Box 3289, 247A Carl Building, Durham, NC 27710. Phone: (919)681-3021, FAX: (919)681-7060. chen0032@ mc.duke.edu. \#Current address: Department of Pediatrics, Sun Yat-Sen Memorial Hospital, Sun Yat-Sen University, Guangzhou, Guangdong, P. R. China

\& Current address: Department of Medicine, Medical University of South Carolina, Charleston, South Carolina, USA. All authors declare no conflict of interest.

Publisher's Disclaimer: This is a PDF file of an unedited manuscript that has been accepted for publication. As a service to our customers we are providing this early version of the manuscript. The manuscript will undergo copyediting, typesetting, and review of the resulting proof before it is published in its final citable form. Please note that during the production process errors may be discovered which could affect the content, and all legal disclaimers that apply to the journal pertain.
} 
Conclusions-IGF-1 mitigates radiation-induced hematopoietic toxicity through protecting hematopoietic stem and progenitor cells from apoptosis and enhancing proliferation and differentiation of the surviving hematopoietic progenitors.

\title{
Keywords
}

Insulin-like growth factor 1; radiation; hematopoiesis; apoptosis; single cell culture

\section{INTRODUCTION}

Radiological accident and terrorism remain a major public health threat (1). The hematopoietic system is one of the most sensitive tissues to radiation toxicity (2). There is currently a lack of countermeasures that can effectively mitigate hematological toxicity after irradiation (2). It was suggested previously that growth hormone mitigates against lethal irradiation through insulin-like growth factor 1 (IGF-1) (3). In addition, it has been demonstrated that IGF-1 decrease apoptosis and promotes hematopoietic progenitor cell survival (4). These biologic features, along with its demonstrated safety profile as indicated by FDA approval in humans (5), make IGF-1 an attractive candidate for use in the treatment of victims of ionizing radiation induced hematopoietic failure. Here, we report the effects of IGF-1 on mitigating hematological toxicity after lethal irradiation.

\section{MATERIAL AND METHODS}

\author{
Mice
}

BALB/c $\left(\mathrm{H} 2^{\mathrm{d}}\right)$ and C57BL/6 $\left(\mathrm{H} 2^{\mathrm{b}}\right)$ were purchased from The Jackson Laboratories (Bar Harbor, Maine). The details about the mice and animal housing were the same as previously described (3).

IGF-1

Recombinant human IGF-1 was purchased from Feldan (Quebec, QC, Canada). For the in vivo studies, IGF-1 was reconstituted in saline and injected into mice intravenously (i.v.) via tail vein or subcutaneously (s.q.). For the in vitro studies, IGF-1 was diluted in tissue culture media.

\section{Irradiation, histology, colony forming unit (CFU) assay, colony forming unit - spleen (CFU- S), flow cytometry}

These assays were performed as described previously (3).

Determination of dose modifying factor (DMF)-The mice were irradiated with several different doses of radiation between 5 to $8.5 \mathrm{~Gy}$. Survival curves were fitted using the logistic model (6) of the form $p(d)-e^{n}(d-L D 50)\left[1+e^{n(d-L D 50)}\right]^{-1}$, where $d$ is the radiation dose, $p(d)$ is the survival probability at dose level d, LD50 is the radiation dose that kills $50 \%$ of animals in 30 days, and $a$ is a fit parameter that adjusts the slope of the survival curve. DMF is the ratio of LD50 estimated in the IGF-1 treated group and the saline group.

\section{Peripheral blood cell counts}

Blood cells were counted in an automatic hematology analyzer (HEMAVET HV950FS, Drew Scientific, Inc., Oxford, CT). 


\section{Isolation of bone marrow nucleated cells and hematopoietic stem cells}

Isolation of bone marrow cells was described previously (3). Lineage-marker negative $\left(\mathrm{Lin}^{-}\right)$cells were isolated via magnetic column purification using the mouse lineage cell depletion kit (Miltenyi Biotec, Auburn, CA). Hematopoietic stem cells (HSC, $\mathrm{CD} 150^{+} \mathrm{CD} 48^{-} \mathrm{CD}^{-} 1^{-}$), multi-potent progenitors (MPP, CD150-CD48 ${ }^{-} \mathrm{CD} 244^{+}$), and restricted progenitors ( $\left.\mathrm{RP}, \mathrm{CD} 150^{-} \mathrm{CD} 48^{+} \mathrm{CD} 244^{+}\right)(7)$ were sorted in a BD FACSVantage SE flow cytometer (Becton Dickinson, San Jose, CA). Dead cells stained with 7-AAD were excluded from analysis and sorting. For the single cell culture experiments, stem cell or progenitor was sorted directly into 96 -well U-bottom plate at 1 cell/well. The presence of a single cell/well was validated under microscope after the plate was sit for 14-16 hours. Only the wells containing 1 cell/well were included in the analyses.

\section{In vitro culture of bone marrow stem cells}

Total bone marrow cells were seeded at $1 \times 10^{5}$ (non-irradiated) or $5 \times 10^{5}$ (irradiated) per well in 24-well plates containing 1ml/well culture medium. Purified HSC, MPP and RP were seeded at 100 cells (non-irradiated) or 200 cells (irradiated) per well in 96-well U bottom plates containing $200 \mu \mathrm{l} /$ well culture medium. The culture system has been described in detail before (3).

\section{Statistical analysis}

Quantitative data are presented as mean \pm SD. Comparison was performed either by Student's t-test (two groups) or analysis of variance (>two groups) for continuous data or by $\log$ rank test for survival data. All statistical analyses were performed using StatView software (SAS institute, Cary, NC) or Microsoft Excel (Microsoft, Seattle, WA). P values less than 0.05 were considered significant.

\section{RESULTS}

\section{IGF-1 mitigates against lethal irradiation}

We first tested the ability of IGF-1 to mitigate against mortality after lethal irradiation. BALB/c mice were whole-body irradiated with $7.5 \mathrm{~Gy}$ and treated with IGF-1 i.v. at a dose of $100 \mu \mathrm{g} / \mathrm{dose} / \mathrm{day}$ once a day for 5 consecutive days starting within one hour after radiation exposure. As shown in Fig. 1a, 8 out of 20 mice (40\%) treated with IGF-1 survived more than 100 days post radiation exposure while only 2 out of 20 mice (10\%) in the control group survived, demonstrating that IGF-1 possesses significant radioprotective effect $(\mathrm{P}<0.05)$. To develop a more practical regimen, we treated the irradiated BALB/c mice with a single dose of IGF-1 s.c. within one hour post-irradiation. As illustrated in Fig. 1b, $100 \mu \mathrm{g}$ of IGF-1 did not significantly protect BALB/c mice from lethal irradiation. An increase of the dose from $100 \mu \mathrm{g}$ to $500 \mu \mathrm{g}$ significantly protected BALB/c mice from lethal irradiation (100-day survival: $60 \%$ vs. $13.3 \%, \mathrm{P}<0.05)$.

Because victims may not be able to receive medical treatment until several hours to days following radiation exposure, it would be useful to determine how long we can wait to treat the animals. As shown in Fig. 1c, IGF-1 treatment could be delayed at least 6 hours (100day survival: $60 \%$ vs. $20 \%, \mathrm{P}<0.01$ ) but not 24 hours.

To determine whether IGF-1 is still effective when higher dose of irradiation is used, we irradiated BALB/c mice with a LD100/30 dose of $8.5 \mathrm{~Gy}$. The mice were then treated with a single s.c. dose of IGF-1 $(500 \mu \mathrm{g})$. As shown in Fig. 1d, IGF-1 still exerted significant radioprotective effects (median survival time: 20 days vs. 13 days, $\mathrm{P}=0.001$ ). 
As shown in Figure 1e, the DMF for 1-dose IGF-1 treatment in BALB/c mice was 1.08 (95\% confidence interval: $1.03-1.13, \mathrm{P}<0.01$ ).

To ascertain that the effects of IGF-1 were not strain specific, we repeated the experiment with C57BL/6 mice. C57BL/6 mice were irradiated with 8.5 Gy and treated with a single dose of IGF-1 (500 $\mu \mathrm{g}$, s.c.) within one hour after radiation exposure. As demonstrated in Fig. 1f, similar to the data obtained from BALB/c mice, IGF-1 was also able to mitigate against lethal irradiation in $\mathrm{C} 57 \mathrm{BL} / 6$ mice $(\mathrm{P}=0.01)$. It is important to point out that all the irradiated mice survived more than 100 days remained healthy for more than one year. When tested more than one year post irradiation, the hematologic and immune parameters remained within the normal range and no tumors were found in all survivors at necropsy (data not shown).

\section{IGF-1 facilitates hematological recovery post-irradiation}

Hematopoietic cells are amongst the most sensitive cell types to ionizing radiation and the mice often die from the sequelae of hematopoietic and immune failure (bleeding and infections) (2). To understand how IGF-1 mitigates against lethal irradiation, we monitored the recovery of white cells, neutrophils, red blood cells, and platelets in peripheral blood following irradiation with $7.5 \mathrm{~Gy}$ at week 2,3 , and 4 . Significant higher numbers of red blood cells and platelets were observed in IGF-1 treated mice compared with the saline control at day +14 (Fig. 2b,c, $\mathrm{P}<0.05$ ). Unfortunately, few control mice survived more than two weeks, preventing us from continuing to compare the differences in hematological recovery at week 3 and 4 .

\section{IGF-1 promotes hematopoietic stem and progenitor cell recovery after irradiation exposure}

To understand how IGF-1 facilitates hematological recovery post lethal irradiation, we studied hematopoietic recovery in bone marrow. Histological analyses of bone marrow suggested that IGF-1 increased the cellularity of bone marrow at day +14 post irradiation (Fig. 3a). Consistent with data from the histological analyses, total bone marrow cells were also increased in the IGF-1-treated group compared with the saline control group (Fig. 3b, $\mathrm{P}<0.01$ ). To further understand the effect of IGF-1 on hematopoiesis, we also measured hematopoietic progenitor cells using a CFU assay. As shown in Fig. 3c-e, IGF-1 significantly increased the numbers of colony forming cells (CFC) in all lineages $(\mathrm{P}<0.05$ in all). Moreover, IGF-1 significantly increased the numbers of $\mathrm{CD} 150^{+} \mathrm{CD} 48^{-} \mathrm{CD} 244^{-} \mathrm{HSC}$ (Fig. 3f, P<0.05).

\section{Effect of IGF-1 on hematopoietic recovery after irradiation in vitro}

To investigate how IGF-1 facilitates hematopoietic recovery after radiation exposure, we adapted an in vitro culture system, in which hematopoietic cells were cultured together with thrombopoietin, stem cell factor, and Flt3 ligand (TSF). This culture system mimics in vivo hematopoiesis and allows stem and progenitor cell expansion as well as differentiation (3). To determine the optimal concentration of IGF-1, C57BL/6 bone marrow cells were irradiated at different doses and cultured with different concentrations of IGF-1. After 7 days in culture, total cell numbers were determined and the degree of cell expansion was presented as fold increase over the original cell numbers plated in the wells. As shown in Fig. 4a, IGF-1 promoted both non-irradiated and irradiated cell expansion at a dosedependent manner up to $1 \mu \mathrm{g} / \mathrm{ml}$. Since $3 \mathrm{~Gy}$ is the highest radiation dose that maintains sufficient cell expansion, this dose of radiation was chosen for the subsequent in vitro experiments. The IGF-1 concentration of $1 \mu \mathrm{g} / \mathrm{ml}$ was also used for the subsequent in vitro studies because this concentration produced the optimal cell expansion. 
We next sought to determine the effects of IGF-1 on hematopoietic stem and progenitor cells using this in vitro culture system. C57BL/6 bone marrow cells were irradiated with 3 Gy and cultured with IGF- $1(1 \mu \mathrm{g} / \mathrm{ml})$ in the presence of TSF. The total cell number, CFC, and CFU-S were determined after 7 days in culture. Similar to the data presented in Fig. 4a, IGF-1 significantly increased the total non-irradiated (Fig. 4b, P<0.01) and irradiated cell number $(\mathrm{P}<0.001)$. Total number of $\mathrm{CFC}$ in both non-irradiated and irradiated bone marrow cells were also significantly increased in the presence of IGF-1 (Fig. $4 \mathrm{~b}, \mathrm{P}<0.05$ ). Total number of CFU-S day 12 (primitive stem cells) in both non-irradiated and irradiated bone marrow cells were not changed in the presence of IGF-1 (Fig. 4b).

To determine whether IGF-1 has a direct effect on irradiated hematopoietic stem or progenitor cells, we repeated the above experiments using purified hematopoietic stem and progenitor cells. As shown in Fig. 4c, IGF-1 significantly increased the total cell number generated by irradiated ( 3 Gy) HSC $(\mathrm{P}<0.05)$, MPP $(\mathrm{P}<0.01)$, as well as $\mathrm{RP}(\mathrm{P}<0.01)$ after 7 days in culture.

\section{IGF-1 protects hematopoietic stem and progenitor cells from apoptosis and death post- irradiation}

The in vitro and in vivo data presented above demonstrate that IGF-1 facilitates the recovery of hematopoietic stem and progenitor cells after radiation exposure. The effects of IGF-1 on hematopoietic recovery could be a result of protection against apoptosis and/or cell death. To determine whether this is the case, we irradiated both whole and $\mathrm{Lin}^{-}$bone marrow cells from C57BL/6 mice at $3 \mathrm{~Gy}$ and cultured them with IGF- $1(1 \mu \mathrm{g} / \mathrm{ml})$ in the presence of TSF. After 24 hours in culture, the percentages of apoptotic and dead cells were determined by flow cytometry after stained with 7-AAD and annexin V (Fig. 5a). As shown in Fig. 5a-c, we did not observe any differences in the percentages of apoptotic and dead cells among total bone marrow cells between IGF-1-treated and control groups. However, the percentages of both apoptotic and dead cells were significantly decreased among $\mathrm{Lin}^{-}$bone marrow cells in IGF-1-treated group compared with the control (Fig. 5a-c, apoptotic: $\mathrm{P}<0.001$, dead: $\mathrm{P}<0.01$ ), demonstrating that IGF-1 protects hematopoietic stem/progenitor cells from radiation-induced apoptosis and cell death.

To validate the protective effects of IGF-1, we developed a single cell culture system. HSC, MPP, and RP were sorted into 96-well U bottom plates at 1 cell/well. The plates were then irradiated at $3 \mathrm{~Gy}$. The presence of a single cell per well was confirmed by light microscopy. This information was recorded and used as a basis to calculate \% positive wells. After cultured with TSF in the presence or absence of IGF-1 $(1 \mu \mathrm{g} / \mathrm{ml})$ for 7 days, the plates were examined by light microscopy and the numbers of wells containing dividing cells were recorded. The percentages of positive wells were calculated by dividing the number of wells containing dividing cells by the number of wells containing a single cell at the initiation of culture. As shown in Fig. 5d, significantly higher percentages of positive wells were observed in HSC, MPP, and RP when IGF-1 was present, further demonstrating that IGF-1 is able to prevent hematopoietic stem and progenitors cells from undergoing apoptosis and death post-irradiation.

\section{Effects of IGF-1 on hematopoietic stem and progenitor cell expansion and differentiation}

Another potential mechanism for IGF-1 to enhance irradiated hematopoietic stem and progenitor cell recovery is through promoting cell expansion and/or differentiation. We used two different culture systems to address this question. In the first culture system, 100 nonirradiated purified stem or progenitor cells were cultured with IGF-1 in the presence of TSF. As shown in Fig. 6a, IGF-1 had no effect on total cell number generated by HSCs after 7 days in culture. A further analysis of the frequencies of CFU-S day 12 and CFC in this 
culture demonstrated that IGF-1 did not impact the stem and progenitor contents (data not shown). By contrast, IGF-1 significantly increased the total cell yields by MPP and RP.

This question was further studied at the single cell level using the same system described in Fig. 5d. As described in detail in Fig. 5d, a single stem or progenitor cell was sorted into each well of a 96-well plate. The numbers of cells per well were counted at day 3,5, and 7 after the initiation of culture. At day 3, the exact number of cells in each well could be counted in all wells. The data from this time point were presented as number of cells per well. After day 5, a significant number of wells had more cells that we could count. The data from day 5 and 7 were presented as \% of wells containing large colonies (HSC: $>100$ cells, MPP: >50 cells, RP: >16 cells). As shown in Fig. 6b, c, IGF-1 has limited effects on expansion and differentiation of both non-irradiated HSC and MPP during 7 days in culture. However, the effects of IGF-1 on RP was significant in both total number of cells at day 3 $(\mathrm{P}<0.05)$ and the percentages of wells containing large colonies $(\mathrm{P}<0.05)$. The single cell culture system also allowed us to study the effects of IGF-1 on cell expansion and differentiation of irradiated stem and progenitor cells without interference with its effects on apoptosis and cell death. Similar to the data obtained in non-irradiated cells, IGF-1 also had limited effects on irradiated HSC and MPP but significant effects on irradiated RP (Fig. 6b, $\mathrm{c}, \mathrm{P}<0.05)$.

\section{DISCUSSION}

In this report, we have demonstrated that IGF-1 mitigates hematopoietic toxicity after lethal dose of total body irradiation (Fig. 1). This effect is not strain specific. One s.q. dose of IGF-1 given after radiation exposure is enough to mitigate and the treatment could be delayed six or more hours after irradiation. These properties offer several practical advantages over other candidate agents in an emergency because IGF-1 could possibly be administered by patients themselves with a window of opportunity of several hours (2).

Because the main target of total body irradiation at the dose that we used is hematopoietic system $(2,3)$, we studied the effect of IGF-1 on hematopoietic recovery post-irradiation. Both in vitro and in vivo data demonstrate that IGF-1 accelerates hematopoietic recovery after irradiation (Fig. 2-4). These data are consistent with the previously published results showing that IGF-1 promotes hematopoiesis (8-10). The immediate effect of ionizing radiation is DNA damage leading to cell apoptosis and death (11). Because it has been demonstrated that IGF-1 prevents apoptosis of hematopoietic progenitors after cytokine withdrawal $(4,12)$, IGF-1 may have the same effect after irradiation. The initial evaluation of total bone marrow cells did not show IGF-1 prevents apoptosis and cell death (Fig. 5a-c). However, further analysis of $\mathrm{Lin}^{-}$cells that contain mostly stem and progenitor cells clearly demonstrate that IGF-1 specifically prevents radiation-induced apoptosis and cell death of hematopoietic stem and progenitor cells (Fig. 5a-c). Importantly, this conclusion was further verified at the single cell level (Fig. 5d).

Another possibility for IGF-1 to accelerate hematopoietic recovery is through promoting expansion and/or differentiation of hematopoietic stem and progenitor cells (13). Data shown in Fig. 4a, b and 6 clearly indicate that IGF-1 promotes expansion and differentiation of progenitor cells but not stem cells. Results from experiments using purified stem and progenitor cells (Fig. 6a) suggest that IGF-1 acts directly on both multipotent and restricted progenitors. To determine whether IGF-1 has a similar effect on irradiated stem and progenitor cells, we developed a single cell culture system so that we could differentiate the effects on apoptosis from cell expansion. The data from these experiments as shown in Fig. $6 \mathrm{~b}, \mathrm{c}$ indicates that IGF-1 promotes expansion and differentiation of restricted progenitor 
cells. IGF-1 may elicit its effects on progenitors through Raf-1 protein as previously reported (14).

In summary, IGF-1 mitigates hematopoietic toxicity after total body irradiation through preventing hematopoietic stem and progenitor cells from undergoing apoptosis and death as well as promoting expansion and differentiation of progenitor cells. Future testing of IGF-1 as a mitigator for radiation injury is warranted.

\section{Acknowledgments}

We thank Dr. John Chute for assistance with the in vitro culture system and Dr. Joel Ross for editing the manuscript. This study was supported by pilot projects funded by NIH grant U19AI067798 (B.J.C.).

\section{References}

1. Hafer N, Cassatt D, Dicarlo A, et al. NIAID/NIH radiation/nuclear medical countermeasures product research and development program. Health Phys. 2010; 98:903-905. [PubMed: 20445403]

2. Waselenko JK, MacVittie TJ, Blakely WF, et al. Medical management of the acute radiation syndrome: recommendations of the Strategic National Stockpile Radiation Working Group. Ann Intern Med. 2004; 140:1037-1051. [PubMed: 15197022]

3. Chen BJ, Deoliveira D, Spasojevic I, et al. Growth hormone mitigates against lethal irradiation and enhances hematologic and immune recovery in mice and nonhuman primates. PLoS One. 2010; 5:e11056. [PubMed: 20585403]

4. Rodriguez-Tarduchy G, Collins MK, Garcia I, et al. Insulin-like growth factor-I inhibits apoptosis in IL-3-dependent hemopoietic cells. J Immunol. 1992; 149:535-540. [PubMed: 1624800]

5. Rosenbloom AL. Mecasermin (recombinant human insulin-like growth factor I). Adv Ther. 2009; 26:40-54. [PubMed: 19198769]

6. Dobson, AJ. An introduction to generalized linear models. 2. Boca Raton: Chapman \& Hall/CRC; 2002.

7. Kiel MJ, Yilmaz OH, Iwashita T, et al. SLAM family receptors distinguish hematopoietic stem and progenitor cells and reveal endothelial niches for stem cells. Cell. 2005; 121:1109-1121. [PubMed: 15989959]

8. Kurtz A, Hartl W, Jelkmann W, et al. Activity in fetal bovine serum that stimulates erythroid colony formation in fetal mouse livers is insulinlike growth factor I. J Clin Invest. 1985; 76:1643-1648. [PubMed: 4056043]

9. Merchav S, Tatarsky I, Hochberg Z. Enhancement of erythropoiesis in vitro by human growth hormone is mediated by insulin-like growth factor I. Br J Haematol. 1988; 70:267-271. [PubMed: 2849979]

10. Sanders M, Sorba S, Dainiak N. Insulin-like growth factors stimulate erythropoiesis in serumsubstituted umbilical cord blood cultures. Exp Hematol. 1993; 21:25-30. [PubMed: 8417957]

11. Hall, EJ.; Giaccia, AJ. Radiobiology for the radiologist. 6. Philadelphia: Lippincott Williams \& Wilkins; 2006.

12. Minshall C, Arkins S, Freund GG, et al. Requirement for phosphatidylinositol 3 '-kinase to protect hemopoietic progenitors against apoptosis depends upon the extracellular survival factor. $\mathrm{J}$ Immunol. 1996; 156:939-947. [PubMed: 8558020]

13. Soon L, Flechner L, Gutkind JS, et al. Insulin-like growth factor I synergizes with interleukin 4 for hematopoietic cell proliferation independent of insulin receptor substrate expression. Mol Cell Biol. 1999; 19:3816-3828. [PubMed: 10207105]

14. Sanders MR, Lu H, Walker F, et al. The Raf-1 protein mediates insulin-like growth factor-induced proliferation of erythroid progenitor cells. Stem Cells. 1998; 16:200-207. [PubMed: 9617895] 


\section{SUMMARY}

IGF-1 administered post-exposure significantly improves survival after lethal total body irradiation through accelerating hematopoietic recovery. IGF-1 protects hematopoietic stem and progenitor cells from radiation-induced apoptosis and cell death. IGF-1 also enhances proliferation and differentiation of the surviving hematopoietic progenitors. 
(a)

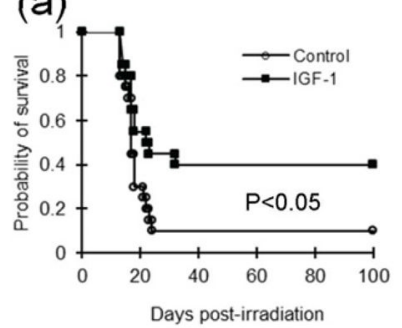

(d)

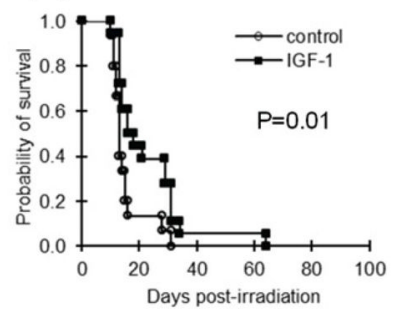

(b)

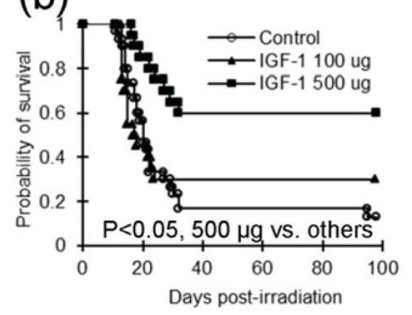

(e)

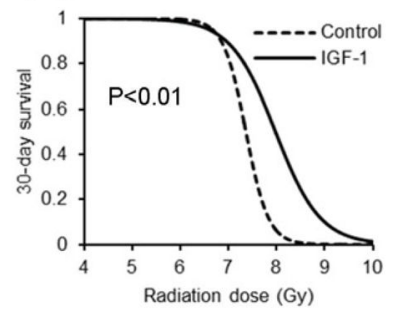

(c)

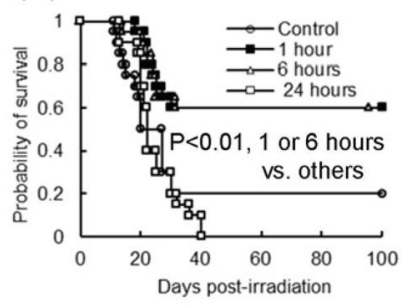

(f)

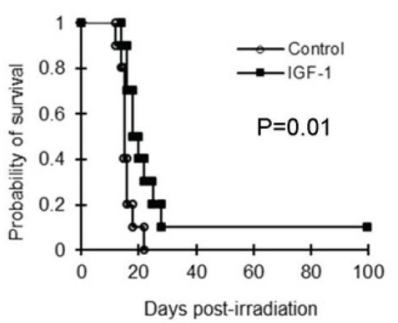

Fig. 1. IGF-1 mitigates against lethal irradiation

Mice were irradiated with different doses of total body radiation (a-c: $7.5 \mathrm{~Gy}$; $\mathrm{d}$ and f: 8.5 Gy; e: 5-8.5 Gy) and treated with IGF-1. Combined results from 2-3 repeats with similar data are presented. Each group contained 10-20 animals. (a) IGF-1 was administered i.v. daily at $100 \mu \mathrm{g} / \mathrm{dose} /$ day for the first 5 days; (b) A single dose of IGF-1 was given by s.q.; (c) A single dose of IGF-1 $(500 \mu \mathrm{g})$ was administered s.q. at different times post irradiation; (d) BALB/c mice were irradiated with $8.5 \mathrm{~Gy}$ and treated with a single s.q. dose $(500 \mu \mathrm{g})$ of IGF-1; (e) DMF; (f) C57BL/6 mice were irradiated with $8.5 \mathrm{~Gy}$ and treated with a single s.q. dose $(500 \mu \mathrm{g})$ of IGF-1. 
(a)

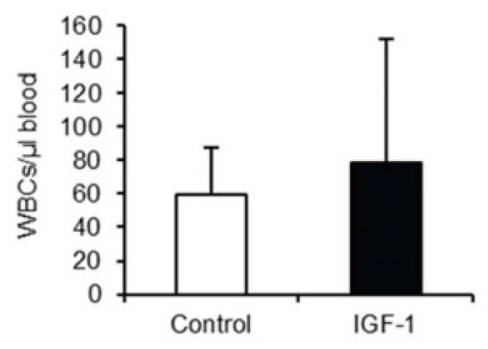

(c)

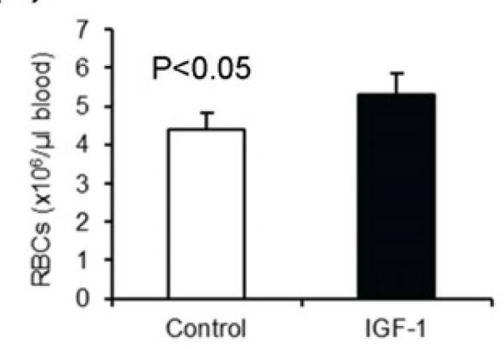

(b)

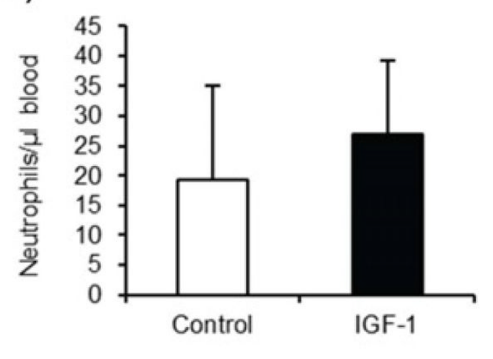

(d)

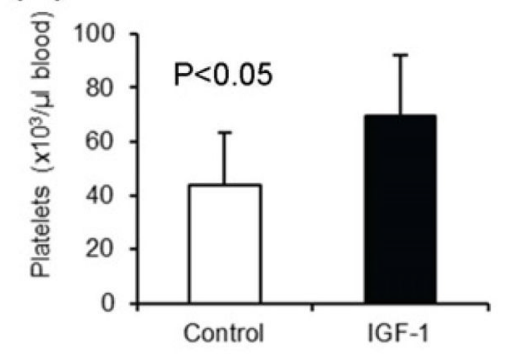

Fig. 2. IGF-1 facilitates hematological recovery post irradiation

BALB/c mice were irradiated with 7.5 Gy. A single dose (500 $\mu \mathrm{g})$ of IGF-1 was administered. Peripheral blood counts $(\mathrm{a}-\mathrm{d})$ were measured at day+14. Each group contained 10 animals. This is a representative of two experiments with similar results. 
(a)

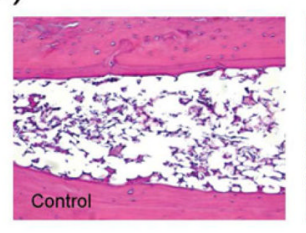

(c)

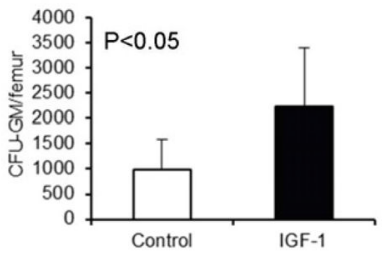

(e)

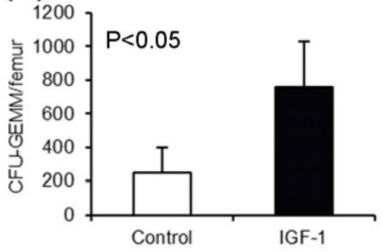

(b)

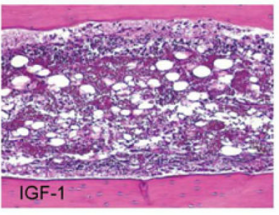

(d)

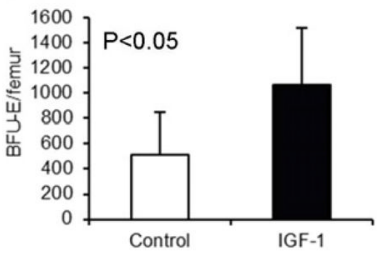

(f)

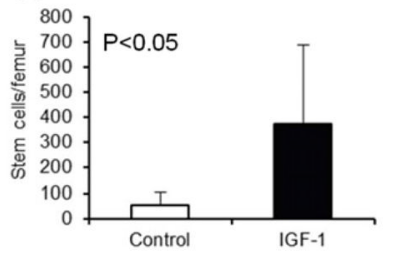

Fig. 3. Effect of IGF-1 on hematopoiesis in vivo

$\mathrm{BALB} / \mathrm{c}$ mice were irradiated with $7.5 \mathrm{~Gy}$ and treated with a single s.c. dose $(500 \mu \mathrm{g})$ of IGF-1. Mice were sacrificed on day +14 . Tibias were harvested and stained with hematoxylin and eosin for histological analyses (a, 12.6x). Femurs were harvested for analysis of cell contents (b-f). Each group contained 7-8 animals. This is a representative of two experiments with similar results. 
(a)

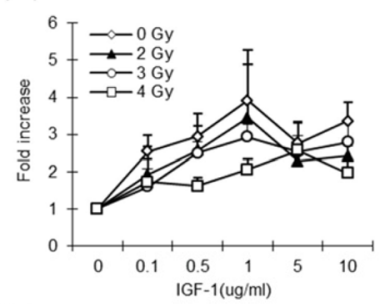

(b)
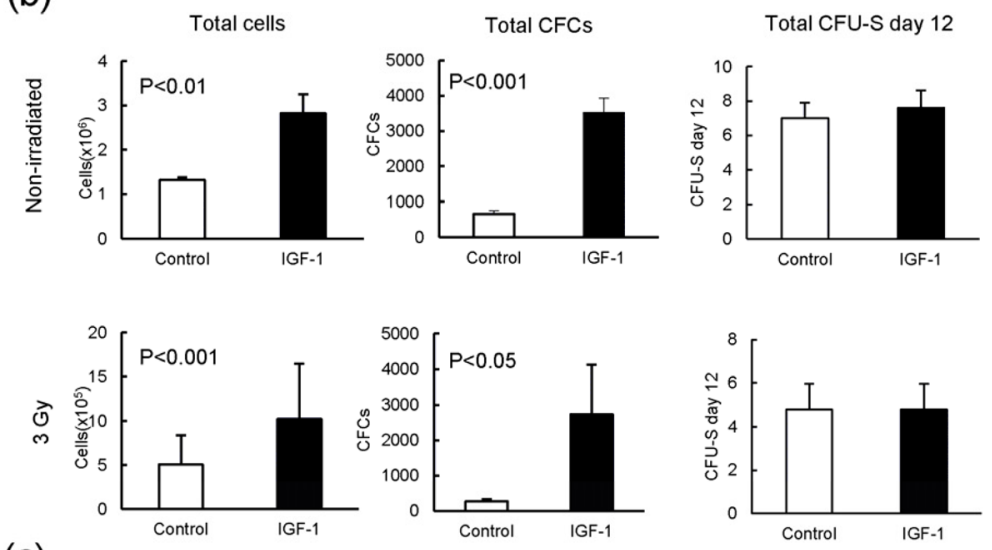

(c)
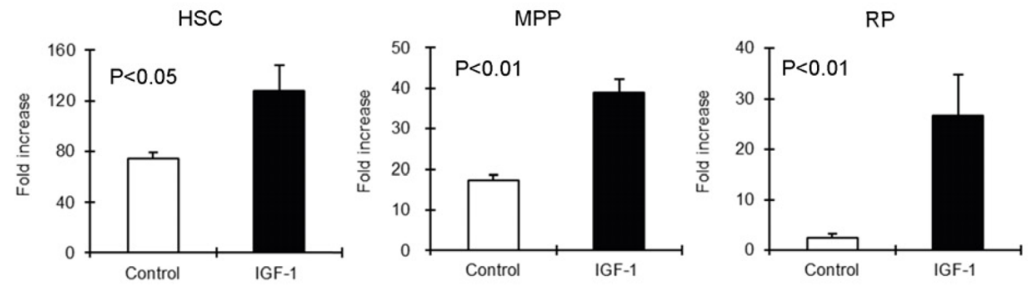

Fig. 4. In vitro effects of IGF-1 on hematopoietic cells post irradiation $1 \times 10^{5}$ non-irradiated or $5 \times 10^{5}$ irradiated bone marrow cells or 100 purified stem or progenitor cells from C57BL/6 mice were cultured in the presence of TSF. The cells were harvested after 7 days in culture. Experiments with similar results have been repeated for at least three times. (a) Titration of radiation dose and IGF- 1 concentration. P<0.05, 0 vs. $1 \mu \mathrm{g}$ at all radiation doses; (b) Numbers of total cells, CFC, and CFU-S day 12; (c) Cell expansion when $3 \mathrm{~Gy}$ irradiated purified hematopoietic stem cells or progenitors were used. 
(a)

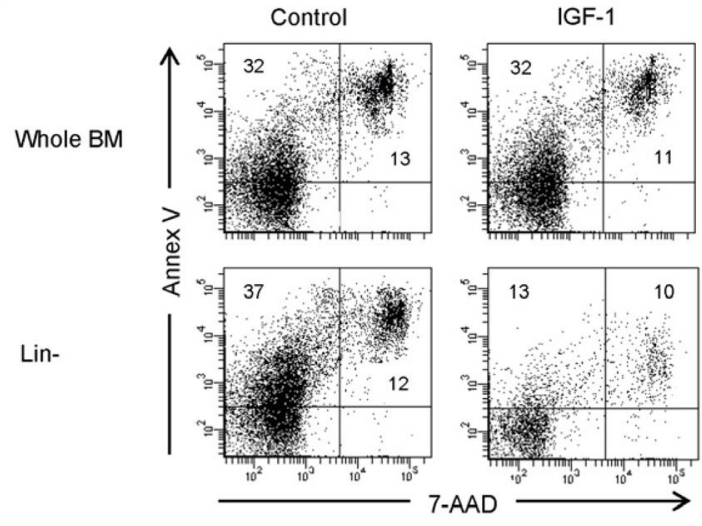

(b)

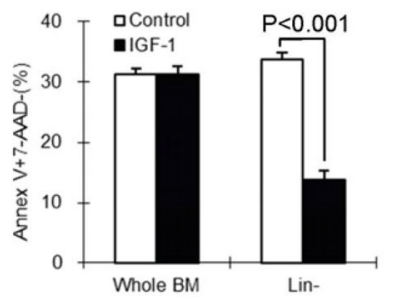

(d)

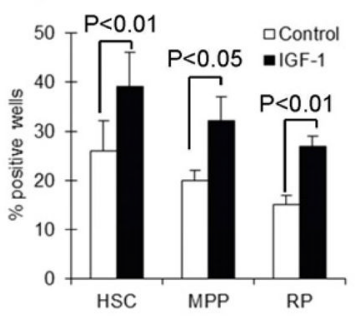

(c)

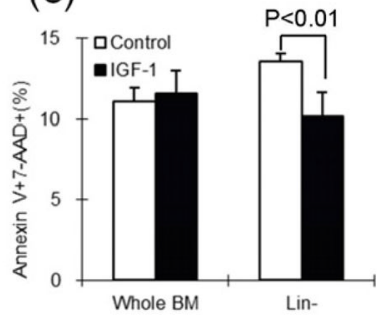

Fig. 5. IGF-1 protects hematopoietic stem and progenitor cells from undergoing apoptosis and death post irradiation

Irradiated (3Gy) whole or subsets of bone marrow cells from C57BL/6 mice were cultured in the presence of TSF. After 24 hours in culture, percentages of apoptotic (Annexin $\mathrm{V}^{+} 7$ $\mathrm{AAD}^{-}, \mathrm{a}$ and $\mathrm{b}$ ) and dead cells (Annexin $\mathrm{V}^{+} 7-\mathrm{AAD}^{+}$, a and $\mathrm{c}$ ) were determined by flow cytometry. In panel d, HSC or MPP or RP were sorted into 96-well round-bottom plate at 1 cell/well. After irradiation, the cells were cultured for 7 days. The results were combined from three experiments with similar results. 
(a)
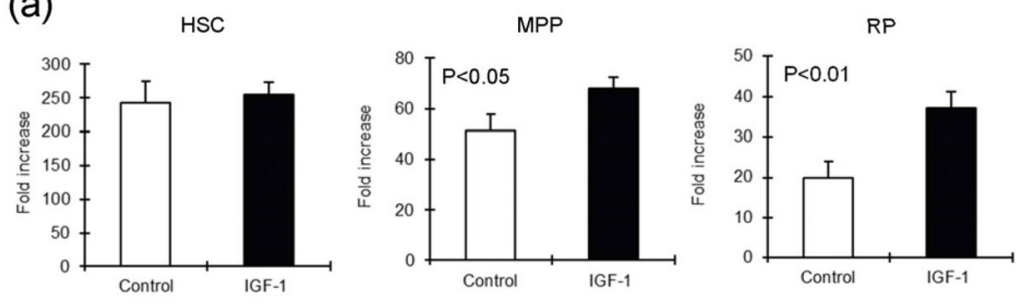

(b)
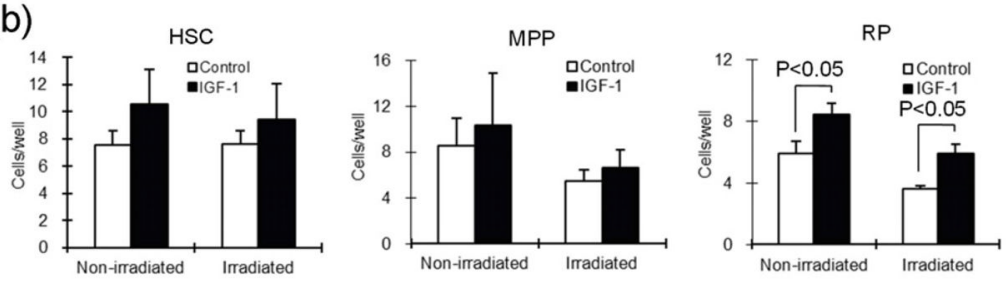

(c)
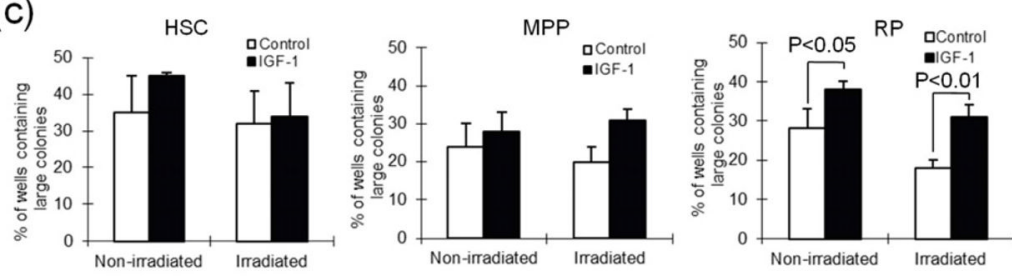

Fig. 6. Effects of IGF-1 on hematopoietic stem and progenitor cell expansion and differentiation Various numbers of non-irradiated $(\mathrm{a}, \mathrm{b}, \mathrm{c})$ or irradiated $(3 \mathrm{~Gy}, \mathrm{~b}$ and $\mathrm{c}$ ) purified stem or progenitor cells from C57BL/6 mice were cultured in the presence of TSF in 96-well Ubottom plates. After 3 (b) or 7 (a and c) days in culture, cell numbers in each well were counted. The results were from at least three experiments with similar results. 